This is a self-archived version of an original article. This version may differ from the original in pagination and typographic details.

Author(s): Lu, Yao; Virtanen, Pauli; Heikkilä, Tero T.

Title: Effect of disorder on Majorana localization in topological superconductors : A quasiclassical approach

Year: 2020

Version: Published version

Copyright: @2020 American Physical Society

Rights: In Copyright

Rights url: http://rightsstatements.org/page/lnC/1.0/?language=en

Please cite the original version:

Lu, Y., Virtanen, P., \& Heikkilä, T. T. (2020). Effect of disorder on Majorana localization in topological superconductors : A quasiclassical approach. Physical Review B, 102(22), Article 224510. https://doi.org/10.1103/physrevb.102.224510 


\title{
Effect of disorder on Majorana localization in topological superconductors: A quasiclassical approach
}

\author{
Yao Lu, Pauli Virtanen, and Tero T. Heikkilä \\ Department of Physics and Nanoscience Center, University of Jyväskylä, P.O. Box 35 (YFL), FI-40014 University of Jyväskylä, Finland
}

(Received 18 May 2020; revised 16 November 2020; accepted 17 November 2020; published 21 December 2020)

Two-dimensional (2D) topological superconductors (TS) host chiral Majorana modes (MMs) localized at the boundaries. In this work, we study the effect of disorder on the localization length of MMs in two-dimensional spin-orbit (SO) coupled superconductors within quasiclassical approximation. We find nonmonotonic behavior of the Majorana localization length as a function of disorder strength. At weak disorder, the Majorana localization length decreases with an increasing disorder strength. Decreasing the disorder scattering time below a crossover value $\tau_{c}$, the Majorana localization length starts to increase. The crossover scattering time depends on the relative magnitudes of the two ingredients behind TS: SO coupling and exchange field. For dominating SO coupling, $\tau_{c}$ is small, and vice versa for the dominating exchange field.

DOI: 10.1103/PhysRevB.102.224510

\section{INTRODUCTION}

Realization of topological superconductors (TSs) supporting Majorana modes (MMs) in condensed matter systems has attracted much attention due to its potential application in quantum computing [1-9]. As random impurities are variantly present in any realistic systems, understanding the effect of disorder on the Majorana localization length is of great importance and interest. It was commonly believed that unlike $s$ wave superconductors, topological superconductors should be treated as effective unconventional superconductors (like $p$ wave superconductors), which violate Anderson's theorem and are very sensitive to disorder. MMs cannot survive when the disorder strength is much larger than the pairing gap, in which case the bulk spectrum becomes gapless.

Plenty of works have been devoted to studying the effect of disorder on MMs in one-dimensional (1D) $p$ wave superconductors [10-17]. It has been shown that disorder reduces the bulk energy gap and increases the localization length of MMs. A phase transition to a topologically trivial phase occurs at the gap closing point where the localization length of MMs diverges. For multichannel systems [18-22], the behavior is similar to the single channel case at weak disorder, but can go through multiple phase transitions at stronger disorder.

Recently, it has been reported that in planar Josephson junctions, which are effectively one-dimensional TSs [23-27], weak disorder can also decrease the Majorana localization length [28]. The low energy physics can be described by a one-dimensional, multiple-channel model. In this model, different channels experience different pairing potentials and the Majorana localization length is determined by the pairing potential with the smallest magnitude. The effect of disorder is to average the pairing potential between the channels. Thus the smallest pairing potential increases and the Majorana localization length decreases.
Two-dimensional (2D) TS supporting chiral Majorana edge modes were theoretically proposed [29-36] and experimentally realized [9] in a quantum anomalous Hall insulator (QAHI) superconductor structure. However, we are not aware of a previous study on the effect of disorder in 2D TSs realized in SO coupled systems. Although the effect of disorder on the chiral Majorana modes has been investigated in $p$ wave superfluids/superconductors [37-40], in SO coupled systems with proximity induced $s$ wave pairing, the results should be different and depend on the ratio between SO coupling and spin-splitting strength.

In this work, we study the properties of MMs in singleband, spin-orbit ( $\mathrm{SO}$ ) coupled superconductors in the presence of weak disorder. SO coupled superconductors subjected to an external magnetic field can be driven to a topological phase and host MMs when an odd number of electron bands are partially occupied. In order to get the spatial distribution of MMs, we adopt the quasiclassical approximation by integrating out the relative momentum in the Green's function. This treatment simplifies the calculations, but we lose the information of the fast oscillating part of the Green's function. However, since we are only interested in the localization length of MMs, the fast oscillating part of the Green's function is not important. At weak disorder, we analytically calculate the Majorana localization length and show that it decreases with increasing disorder strength for any SO coupling strength and exchange field. This effect of disorder is due to a renormalization of the Fermi velocity. We also numerically solve the Eilenberger equation and get the localization length for an arbitrary disorder. We find that the Majorana localization length starts to increase with an increasing disorder strength when the disorder scattering time becomes shorter than a crossover scattering time $\tau_{c}$. This crossover scattering time vanishes in the strong SO limit and increases monotonically when increasing the exchange field. 


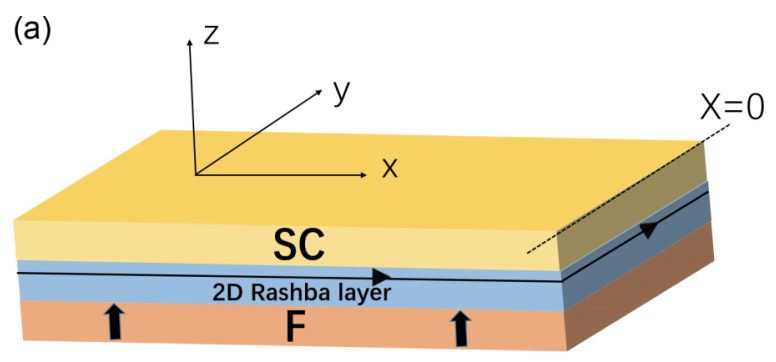

(b)

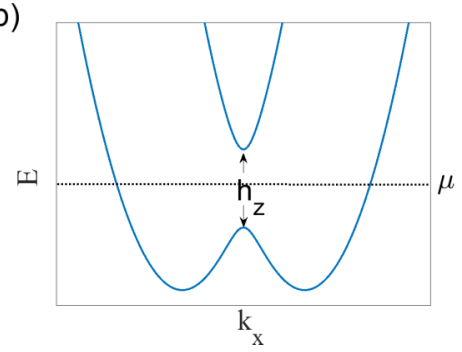

FIG. 1. (a) Sketch of the system under consideration. 2D Rashba layer sandwiched by a superconducting thin film and a ferromagnetic insulator. (b) Schematic picture of the band structure. The chemical potential only cuts the lower band, so that the system is in the topological phase.

\section{MODEL HAMILTONIAN}

We consider a heavy metal thin film with strong SO coupling sandwiched by a superconducting thin film and a ferromagnetic insulator as shown in Fig. 1(a). The effective Hamiltonian describing the 2D Rashba layer with proximity induced pairing and exchange field is given by

$$
H=\int d \boldsymbol{r} \Psi^{\dagger}(\boldsymbol{r}) H_{0} \Psi(\boldsymbol{r})+\Delta c_{\uparrow}^{\dagger}(r) c_{\downarrow}^{\dagger}(r)+\text { h.c. },
$$

with

$$
H_{0}=\frac{-\nabla^{2}}{2 m}-\mu-i \alpha\left(\nabla_{x} \sigma_{2}-\nabla_{y} \sigma_{1}\right)+h_{z} \sigma_{3}+U(\boldsymbol{r}) .
$$

Here $\Psi(\boldsymbol{r})=\left[c_{\uparrow}(\boldsymbol{r}), c_{\downarrow}(\boldsymbol{r})\right]^{\mathrm{T}}$, where $c_{s}(\boldsymbol{r})^{\dagger}$ is the creation operator which creates one electron at position $\boldsymbol{r}$ with spin $s$. $m, \mu$ and $\Delta$ denote the effective mass, chemical potential, and pairing potential, respectively. $\alpha$ is the SO coupling coefficient and $h_{z}$ is the exchange field in the out-of-plane direction. $U(\boldsymbol{r})$ is the Gaussian disorder potential with the correlator $\left\langle U(\boldsymbol{r}) U\left(\boldsymbol{r}^{\prime}\right)\right\rangle=\delta\left(\boldsymbol{r}-\boldsymbol{r}^{\prime}\right) / \pi n \tau$, where $\tau$ is the scattering time of particles in the disordered system and $n$ is the density of states per unit cell at the Fermi level. The schematic band structure (without disorder and superconductivity) is shown in Fig. 1(b). Here we consider the case where the chemical potential only cuts the lower band, so that the system is in the topological phase and hosts chiral Majorana edge states [5]. Taking into account the effect of disorder and expressing it in spin $\otimes$-hole space, the Gorkov equation is given by

$$
\left(G_{0}^{-1}+\mu-\hat{\Sigma}\right) G=1
$$

with

$$
\hat{G}_{0}^{-1}=-\frac{\boldsymbol{k}^{2}}{2 m_{N}}-\left(\alpha k_{x} \sigma_{2}-\alpha k_{y} \sigma_{1}\right)+\left(\epsilon-h_{z} \sigma_{z}\right) \tau_{z} .
$$

Here $\sigma_{i}$ and $\tau_{i}$ are Pauli matrices acting on spin and particlehole space, respectively. $\hat{\Sigma}=\frac{\langle G\rangle}{2 \tau}$ is the disorder self-energy, where $\langle\cdot\rangle$ means an average over all momenta. To investigate the properties of MMs, we assume the system is in the region $x<0$. We use periodic boundary conditions in the $y$ direction and study the Majorana edge states localized on the $x=0$ edge.

\section{QUASICLASSICAL APPROXIMATION}

A generalized quasiclassical theory can be obtained by projecting the Gorkov-Green's function onto the lower band [41-43]. The resulting Eilenberger equation is given by (see Appendix A)

$$
\boldsymbol{v}_{F} \cdot \hat{\nabla} \hat{g}_{\boldsymbol{n}_{F}}=\left[\hat{g}_{\boldsymbol{n}_{F}}, i \epsilon \tilde{\tau}_{3}+\Delta \tilde{\tau}_{1}+\hat{\Sigma}\right],
$$

where $\tilde{\tau}_{i}$ is the Pauli matrix acting on projected space and $\hat{g}_{n_{F}}$ is the quasiclassical Green's function defined by

$$
\hat{g}_{\boldsymbol{n}_{F}}(\epsilon ; \boldsymbol{R})=\int \frac{d \epsilon_{p}}{i \pi} \hat{G}(\epsilon ; \boldsymbol{R}, \boldsymbol{p}) .
$$

The disorder self-energy in the Born approximation becomes $\hat{\Sigma}=\frac{\langle\hat{g}\rangle_{n_{F}}}{2 \tau}$, where $\tau$ is the disorder scattering time. Here $\boldsymbol{n}_{F}$ is the unit vector along the direction of Fermi momentum $\boldsymbol{p}_{F}$ and $\langle\cdot\rangle_{n_{F}}$ means an angular average over all the momentum directions. This angular average should be done in the usual spin $\otimes$ particle-hole space. After we get the self-energy we project it back onto the lower-band subspace. The Eilenberger equation is supplemented by the normalization condition $\hat{g}^{2}=I_{P}$, where $\hat{I}_{P}$ is the identity operator in the lower-band subspace. Writing $\hat{g}$ in terms of Pauli matrices $\hat{g}=g_{1} \tilde{\tau}_{1}+g_{2} \tilde{\tau}_{2}+\hat{g}_{3} \tilde{\tau}_{3}$, the normalization condition becomes $g_{1}^{2}+g_{2}^{2}+g_{3}^{2}=1$. In the clean limit $\hat{\Sigma}=0$, solving Eq. (5) yields

$$
\begin{aligned}
& g_{\boldsymbol{n}_{F}, 1}=\frac{\Delta}{\sqrt{\Delta^{2}-\epsilon^{2}}}-\frac{\epsilon}{\sqrt{\Delta^{2}-\epsilon^{2}}} A e^{\kappa x}, \\
& g_{\boldsymbol{n}_{F}, 2}=\lambda A e^{\kappa x}, \\
& g_{\boldsymbol{n}_{F}, 3}=\frac{i \epsilon}{\sqrt{\Delta^{2}-\epsilon^{2}}}-\frac{i \Delta}{\sqrt{\Delta^{2}-\epsilon^{2}}} A e^{\kappa x} .
\end{aligned}
$$

Here, $\kappa=\frac{2 \sqrt{\Delta^{2}-\epsilon^{2}}}{v_{F} \cos (\phi)}$, where $\phi$ is the angle between $\boldsymbol{n}_{F}$ and the $x$ axis. $\lambda$ denotes the sign of the $x$ component of $\boldsymbol{n}_{F}$. $A$ is a constant determined by the boundary conditions. The boundary condition for an Eilenberger equation is given by $[44,45]$

$$
\hat{g}_{\overline{\boldsymbol{n}}_{F}}=\hat{R} \hat{g}_{\boldsymbol{n}_{F}} \hat{R}^{\dagger}
$$

where $\boldsymbol{n}_{F}$ and $\overline{\boldsymbol{n}}_{F}$ are two momentum directions with the same $y$ components but opposite $x$ components. In the presence of translational invariance in the $y$ direction, an electron with momentum in $\boldsymbol{n}_{F}$ direction is reflected back into an electron with momentum in $\overline{\boldsymbol{n}}_{F}$ direction. $R$ is the reflection part of the scattering matrix at the boundary, and has the form

$$
\hat{R}=\left[\begin{array}{cc}
e^{i \theta} & 0 \\
0 & e^{-i \theta}
\end{array}\right] e^{i \gamma}
$$

The overall phase factor $e^{i \gamma}$ does not affect the solution of the Eilenberger equation and we drop it in the rest of the paper. For a conventional $s$ wave superconductor $\theta=0$ and $A=0$, 
so that the quasiclassical Green's function is homogeneous and there are no edge states. Solving the scattering problem for Eq. (4), we find (see Appendix B)

$$
\theta=\arg (\sin \phi-i X \cos \phi),
$$

where $S_{F}=\sqrt{\alpha^{2} p_{F}^{2}+h_{z}^{2}}$ and $X$ is the time reversal symmetry breaking factor defined by $X=\frac{h_{z}}{S_{F}}$. Matching the boundary conditions at $x=0$, we get

$$
A=\frac{\Delta \tan \theta}{\sqrt{\Delta^{2}-\epsilon^{2}}+\epsilon \tan \theta} .
$$

The density of states $N(\epsilon, x)$ is the real part of $g_{3}$ times the normal state density of states $1 / \pi v_{F}$

$N(\epsilon, x)=2 \frac{\Re g_{3}\left(\epsilon+i 0^{+}\right)}{\pi v_{F}}=\frac{2 \sqrt{\Delta^{2}-\epsilon^{2}}}{v_{F} \cos \phi} \delta(\epsilon+\Delta \cos \theta) e^{\kappa x}$.

From this expression, one can see that there is a low energy quasiparticle excitation localized at the edge. This is the Majorana mode. The energy dispersion of Majorana edge states can be read out from the delta function

$$
\epsilon=-\Delta \cos \theta=\frac{\operatorname{sgn}(X) \Delta \sin \phi}{\sqrt{\sin ^{2} \phi+\frac{h_{z}^{2} \cos ^{2} \phi}{S_{F}^{2}}}}=\frac{\operatorname{sgn}(X) \Delta p_{y}}{p_{F} \sqrt{1-\frac{\alpha^{2}\left(p_{F}^{2}-p_{y}^{2}\right)}{S_{F}^{2}}}} .
$$

As the scattering phase shifts only depend on the momentum direction relative to the interface, and the quasiclassical boundary conditions are generally local, the edge state is present on all vacuum boundaries of the system, regardless of their orientation, as long as they are flat on the scale of the Fermi wavelength.

At low energy, the group velocity of the Majorana mode is given by

$$
v_{M}=\frac{\partial \epsilon}{\partial p_{y}} \approx \frac{\Delta}{p_{F} X} .
$$

Using the same method, we obtain the group velocity of the edge mode on the other edge $v_{M}^{\prime} \approx-\frac{\Delta}{p_{F} X}$, indicating that the edge mode is chiral and propagates in one direction. The localization length of the zero energy Majorana mode is $l_{M}=$ $v_{F} / \Delta$. Integrating $N(\epsilon, x)$ over $x$, we get the total density of states

$$
N(\epsilon)=\int d x N(\epsilon, x)=\delta(\epsilon+\Delta \cos \theta),
$$

which shows that the edge mode is indeed a single channel mode. One interesting property of this chiral Majorana mode is that the number of low energy states depends on $X$. According to $\epsilon=-\Delta \cos \theta$, a low energy edge state corresponds to a large $\theta$. When $X \ll 1, \theta$ is finite only when $\phi$ is small. Thus, there is only a small number of low energy states, and the group velocity of the chiral Majorana mode is large [Fig. 2(a)]. In the opposite limit, when $X \approx 1, \theta$ is finite for a wide range of $\phi$, which indicates that there are a large number of low energy modes, each with a small group velocity [Fig. 2(b)]. For $X \rightarrow 1$, this model becomes similar to the spinless chiral $p$ wave superfluid [37-40]. Below we show that this property
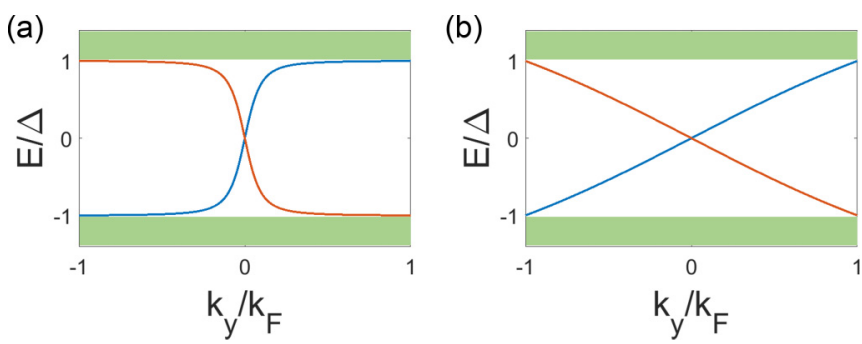

FIG. 2. Energy spectrum of subgap states without disorder calculated from Eq. (13). Red and blue lines denote edge states on different edges and the green area denotes continuous spectrum of the bulk. (a) $X=0.1$, there is only a small number of low energy edge states. (b) $X=0.9$, there are more low energy states.

is useful for understanding the effect of strong disorder on the Majorana localization length.

\section{EFFECT OF DISORDER ON MAJORANA LOCALIZATION LENGTH}

In the presence of the disorder potential, we need to add the self-energy term $\hat{\Sigma}$ to the Eilenberger equation. Here we consider the weak disorder case and treat disorder potential as a perturbation. Then we can approximate the disorder selfenergy as $\hat{\Sigma}=\left\langle\hat{g}^{0}\right\rangle_{\boldsymbol{n}_{F}} / 2 \tau$, where $\hat{g}^{0}$ is the Green's function without disorder given by Eq. (7). For convenience we separate the "bulk" part and the "edge" part of the Green's function without disorder

$$
\hat{g}=\hat{g}_{B}+A \hat{g}_{E} e^{\kappa x},
$$

where $\hat{g}_{B}$ is homogeous, describing the bulk properties, and $\hat{g}_{E}$ is proportional to the exponential factor $e^{\kappa x}$, describing the properties of edge states. They are given by

$$
\begin{gathered}
\hat{g}_{B}=\frac{\Delta}{\sqrt{\Delta^{2}-\epsilon^{2}}} \tilde{\tau}_{1}+\frac{i \epsilon}{\sqrt{\Delta^{2}-\epsilon^{2}}} \tilde{\tau}_{3} . \\
\hat{g}_{E}=-\frac{\epsilon}{\sqrt{\Delta^{2}-\epsilon^{2}}} \tilde{\tau}_{1}+\tau_{2}-\frac{i \Delta}{\sqrt{\Delta^{2}-\epsilon^{2}}} \tilde{\tau}_{3} .
\end{gathered}
$$

Similarly, the self-energy can be written as

$$
\hat{\Sigma}=\hat{\Sigma}_{B}+\hat{\Sigma}_{E} e^{\kappa x},
$$

where $\hat{\Sigma}_{B}$ is homogeneous and $\hat{\Sigma}_{E} e^{\kappa x}$ decays exponentially away from the boundary. Since we are studying the localization length of the zero energy state, we focus on the Green's function with $\boldsymbol{n}_{F}$ pointing to the positive $x$ direction denoted as $\hat{g}^{+}$. The self-energy enters the Eilenberger equation in the commutator, which is

$$
\begin{aligned}
{\left[\hat{\Sigma}, \hat{g}^{+}\right]=} & {\left[\hat{\Sigma}_{B}+\hat{\Sigma}_{E} e^{\kappa x}, \hat{g}_{B}^{+}+A \hat{g}_{E}^{+} e^{\kappa x}\right] } \\
= & {\left[\hat{\Sigma}_{B}, \hat{g}_{B}^{+}\right]+\left(\left[\hat{\Sigma}_{E}, \hat{g}_{B}^{+}\right]+A\left[\hat{\Sigma}_{B}, \hat{g}_{E}^{+}\right]\right) e^{\kappa x} } \\
& +A\left[\hat{\Sigma}_{E}, \hat{g}_{E}^{+}\right] e^{2 \kappa x} .
\end{aligned}
$$

Since we are only interested in the Majorana localization legnth, we focus on the Green's function far away from the boundary, where $\hat{g}_{E}$ and $\Sigma_{E}$ can be treated as perturbations. Thus, we can drop the third term on the right hand side of Eq. (20), which is a higher order perturbation. Note that in the second term on the right-hand side of Eq. (20), A 
is divergent, such that we can ignore the $\hat{\Sigma}_{E} \hat{g}_{B}$ term. Then it can be seen that $\hat{\Sigma}_{E}$ does not appear in the Eilenberger equation. The disorder self-energy has only a bulk contribution $\hat{\Sigma}_{B}=\left\langle\hat{g}_{B}\right\rangle_{\boldsymbol{n}_{F}} / 2 \tau$. Here we need to be careful with the angular average since the basis $\tilde{\tau}_{i}$ is angular dependent. At weak disorder, the self-energy is given by [42]

$$
\hat{\Sigma} \approx \hat{\Sigma}_{B}=\left(\frac{1}{2} \hat{g}_{B}+\frac{X^{2}}{2} \tilde{\tau}_{3} \hat{g}_{B} \tilde{\tau}_{3}\right) / \tau .
$$

Substituting Eq. (21) into Eq. (5), we obtain the Eilenberger equation in the presence of weak disorder

$$
\boldsymbol{v}_{F}^{\prime} \cdot \hat{\nabla} \hat{g}_{\boldsymbol{n}_{F}}=\left[\hat{g}_{\boldsymbol{n}_{F}}, i \in \tilde{\tau}_{3}+\Delta^{\prime} \tilde{\tau}_{1}\right] .
$$

This Eilenberger equation has exactly the same structure as that in the clean case, but with a renormalized Fermi velocity and pairing potential, which are given by

$$
\begin{aligned}
& v_{F}^{\prime}=\frac{v_{F}}{1+\frac{1}{2 \tau \Delta}\left(1+X^{2}\right)} \\
& \Delta^{\prime}=\frac{\Delta\left[1+\frac{1}{2 \tau \Delta}\left(1-X^{2}\right)\right]}{1+\frac{1}{2 \tau \Delta}\left(1+X^{2}\right)} .
\end{aligned}
$$

It can be seen that both Fermi velocity and pairing potential are reduced by disorder. The Majorana localization length is thus

$$
l_{M}=\frac{v_{F}^{\prime}}{\Delta^{\prime}}=\frac{v_{F}}{\Delta\left[1+\frac{1}{2 \tau \Delta}\left(1-X^{2}\right)\right]} .
$$

Since $X^{2}<1, l_{M}$ is always smaller than $l_{M 0}=v_{F} / \Delta$, which is the Majorana localization length in the clean case. Weak disorder thus reduces the Majorana localization length for any SO coupling strength and exchange field. This effect is opposite to that in one dimension, where weak disorder usually increases the Majorana localization length [10-16]. The main difference between $2 \mathrm{D}$ and $1 \mathrm{D}$ systems is that in two dimensions there are many states near the Fermi energy and only a few of them contribute to the Majorana edge states. Hence, at weak disorder the disorder self-energy has only a bulk contribution. However, in one dimension, there are only two channels near the Fermi energy, both of which contribute to the Majorana end states. Thus, the edge contribution to the self-energy has a large impact on the Majorana localization length.

\section{MAJORANA LOCALIZATION LENGTH FOR ARBITRARY DISORDER STRENGTH}

In order to obtain the Majorana localization length for an arbitrary disorder strength, we numerically solve Eq. (5) (Appendix D). Here we use an exponential function DOS = $A e^{-\kappa x}$ to fit the tail of the spatial dependent density of states, and the Majorana localization length $l_{M}$ is defined as $l_{M}=$ $2 / \kappa$. The result is shown in Fig. 3. It can be seen that weak disorder decreases $l_{M}$ for $X=0.8,0.6,0.4$. Increasing the disorder strength, the Majorana localization length starts to increase after the disorder strength reaches the crossover value $1 / \tau_{c}$. The crossover disorder strength depends on $X$. In particular, $1 / \tau_{c}$ goes to zero when $X$ approaches 1 , and increases monotonically with decreasing $X$.

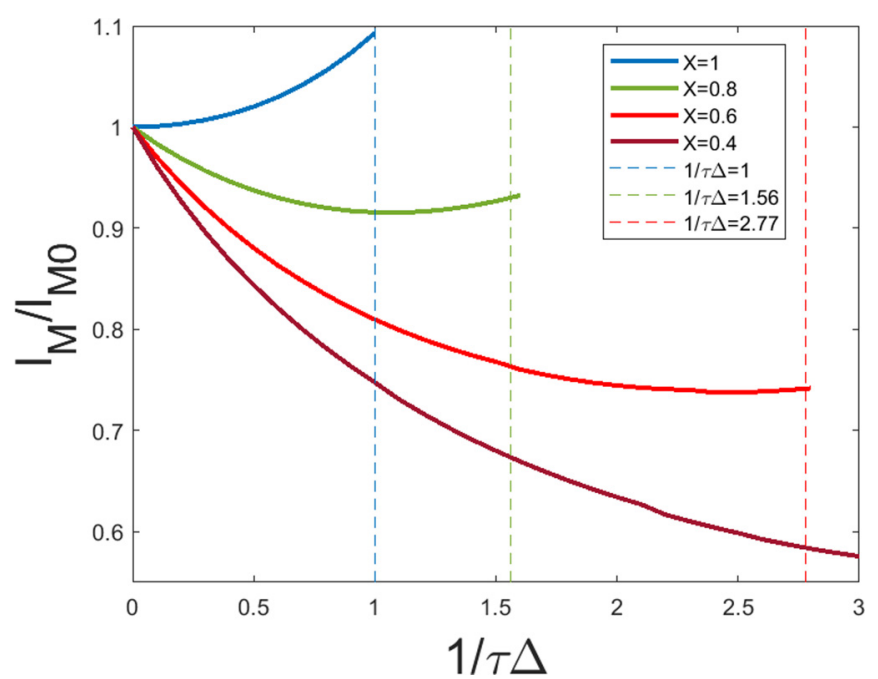

FIG. 3. Majorana localization length $l_{M}$ versus disorder strength for different time reversal symmetry breaking factors $X=1,0.8$, $0.6,0.4$. The vertical dashed lines label the gap closing points. At weak disorder, $l_{M}$ decreases with increasing $1 / \tau$, while at large disorder $l_{M}$ increases with increasing $1 / \tau$. The crossover disorder $1 / \tau_{c}$ is much smaller than pairing $1 / \tau_{c} \ll \Delta$ for $X=1$, and increases when increasing $X$. Here Majorana localization length is normalized by $l_{M 0}$ and the disorder strength is normalized by the order parameter $\Delta$.

To understand the behavior of $\tau_{c}$, we note that the increase of $1 / \tau_{c}$ is caused by the edge self-energy $\hat{\Sigma}_{E}$. For small $X$, as mentioned above, the number of edge states is small (Fig. 2), and thus a large disorder strength is required to increase $l_{M}$. Thus, the crossover disorder is large. Note that near the gap closing point $1 / \tau=\Delta / X^{2}$ (Appendix E), the Majorana localization length is finite, unlike in the $1 \mathrm{D}$ case where the Majorana localization length is divergent near the gap closing point. We explain this in Appendix F. We also calculate the crossover disorder as a function of $X$ as shown in Fig. 4. It shows that when $X$ is close to 1 , the crossover disorder scales linearly with $1-X$.

The physical picture of the nonmonotonic behavior of the localization length is the following. Disorder affects the Majorana localization length in two ways. On one hand, disorder decreases the electron group velocity, which makes the Majorana modes more localized. On the other hand, disorder reduces the quasiparticle spectrum gap and delocalizes the Majorana modes. At weak disorder, the former effect is important, vice versa the latter is important at strong disor-

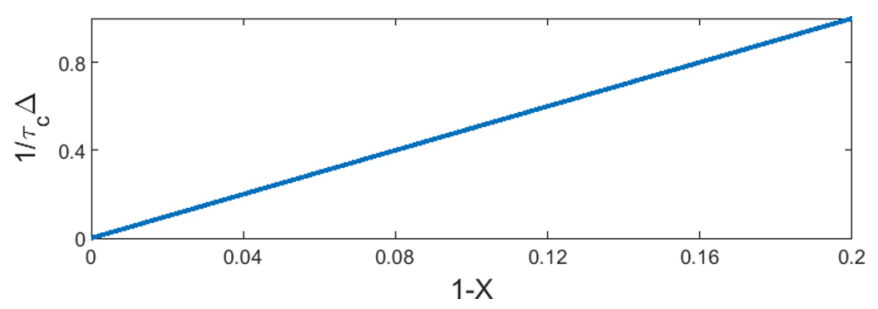

FIG. 4. Crossover disorder strength $1 / \tau_{c} \Delta$ versus $1-X .1 / \tau_{c} \Delta$ depends linearly on $1-X$ for $X \approx 1$. 


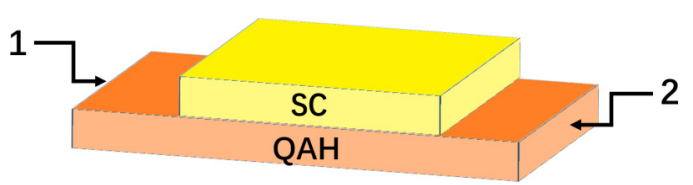

FIG. 5. Sketch of QAHI-superconductor structure in the experiment. The longitudinal conductance $\sigma_{12}$ is measured.

der leading to the non-monotonic behavior of the Majorana localization length. At large disorder strength, the system is expected to undergo a transition at which the localization length diverges. Such transitions in 2D class-D systems can occur when the DOS already has a soft gap [46], and so closing of the bulk gap in the Born approximation is not necessarily correlated with the transition [47].

\section{RELATION TO THE EXPERIMENT}

Recently, a two-dimensional topological superconductor has been realized in a quantum anomalous Hall insulator (QAHI)-superconductor structure [9] as shown in Fig. 5. In the topological phase, the longitudinal conductance is halfquantized $\sigma_{12}=e^{2} / 2 h$ as a signature of the chiral Majorana edge state. The effective Hamiltonian describing the QAHI in the ultrathin limit is given by $[30,48]$.

$$
H_{\mathrm{QAHI}}=D \boldsymbol{k}^{2}+\left(M+B \boldsymbol{k}^{2}\right) \sigma_{z}+A\left(k_{x} \sigma_{x}+k_{y} \sigma_{y}\right)-\mu .
$$

Although this Hamiltonian is different from Eq. (2) discussed in this work, in the case where the chemical potential cuts the conduction band, these two systems both have a nondegenerate Fermi surface with the same spin texture. So the QAHI can be described by a similar quasiclassical theory with a modified boundary condition (Appendix B). Here the time reversal symmetry breaking factor is defined by $X=$ $\frac{h_{\text {eff }}}{\sqrt{h_{\text {eff }}^{2}+A^{2} p_{F}^{2}}}$, where $h_{\text {eff }}$ is the effective exchange field $h_{\text {eff }}=$ $M+B p_{F}^{2}$. Using realistic parameters [48], we find that in QAHI the SO coupling strength and exchange field are of the same order, or $X$ is around 0.7 .

In a system with finite width, the measured conductance might not be precisely quantized due to the coupling between Majorana states on different edges. We predict that in this case weak disorder can shorten the Majorana localization length and make the measured conductance more precisely quantized. In systems where the surface is accessible to scanning tunneling microscopy (STM) measurements, the spatial extent of the Majorana states can also be probed via the local density of states visible in STM.

\section{DISCUSSION AND CONCLUSION}

In conclusion, we use quasiclassical theory to study the effect of disorder on the Majorana localization in a two-dimensional topological superconductor. We find the nonmonotonic behavior of the Majorana localization length $l_{M}$ as a function of disorder strength. We show that weak disorder decreases $l_{M}$ while strong disorder increases it. The crossover disorder strength $1 / \tau_{c}$, where $\left.\frac{d l_{M}}{d \tau}\right|_{\tau=\tau_{c}}=0$, depends on the time reversal symmetry breaking factor $X=h_{z} / S_{F}$.
$1 / \tau_{c}$ decreases toward zero when $\left|h_{z}\right| \ll \alpha p_{F}$, and increases when reducing $X$.

The fact that disorder can decrease Majorana localization length was first reported in one-dimensional multi-channel superconductors [28]. In our work, the physics is different from [28]. In our case, the chemical potential only cuts one band in the normal state, and the decreased Majorana localization length is attributed to the renormalized Fermi velocity. Although in this work we study a specific model, our results are valid in any two-dimensional gapped topological superconductors. This is because the renormalization of the Fermi velocity is universal in two-dimensional superconductors, but the time reversal symmetry breaking factor $X$ has different expressions in different models [9] depending on the type of SO coupling and the direction of the magnetic field.

\section{ACKNOWLEDGMENT}

This work is supported by the Academy of Finland project HYNEQ (Project No. 317118).

\section{APPENDIX A: BASIS OF THE PROJECTED EILENBERGER EQUATION}

Since the chemical potential cuts only the lower band, we can ignore the high energy band and project the Eilenberger equation onto the lower band. The eigenvector of the lower band used here is

$$
\begin{aligned}
& \left|\psi_{-, e}\right\rangle=\left(\alpha p_{F} e^{i \phi^{\prime} / 2}, \quad\left(S_{F}+h_{z}\right) e^{-i \phi^{\prime} / 2}, \quad 0, \quad 0\right)^{\mathrm{T}} / N, \\
& \left|\psi_{-, h}\right\rangle=\left(\begin{array}{lll}
0, & 0, & \left.\left(S_{F}+h_{z}\right) e^{i \phi^{\prime} / 2}, \quad \alpha p_{F} e^{-i \phi^{\prime} / 2}\right)^{\mathrm{T}} / N,
\end{array}\right.
\end{aligned}
$$

where $\left|\psi_{-, e}\right\rangle$ and $\left|\psi_{-, h}\right\rangle$ are electron and hole parts of the eigenvector, respectively. $N$ is the normalization factor $N=$ $\sqrt{2 S_{F}\left(S_{F}+h_{z}\right)} . \phi^{\prime}$ is the angle between the momentum direction and the $y$ axis.

\section{APPENDIX B: SPECULAR HARD-WALL SCATTERING}

The quasiclassical boundary condition [Eq. (8)] is expressed in terms of the scattering matrix of the interface [45]. To find it, we solve here the specular hard-wall scattering problem for Eq. (4) in the normal state, for the $2 \times 2$ electron and hole blocks $H_{\tau}=\left.\left(\epsilon \tau_{z}-\mu-\hat{G}_{0}^{-1}\right)\right|_{\tau_{z} \mapsto \tau= \pm 1}$. The bulk material resides at $x<0$ and is terminated by the boundary at $x=0$. Due to the exchange field in the bulk, the reflection phase is not necessarily the same for electrons and holes, and needs to be calculated explicitly.

We assume $\mu$ is such that there is a single Fermi surface on the lower helical band. The scattering wave function at $x \leqslant 0$ is

$$
\Psi_{\tau}(x)=e^{i k_{\tau} x} \chi_{\mathrm{i}, \tau}+r_{\tau} e^{-i k_{\tau} x} \chi_{\mathrm{o}, \tau}+c_{\tau} e^{\kappa_{\tau} x} \chi_{\mathrm{ev}, \tau},
$$

where $\chi_{\mathrm{i}, \mathrm{o}, \mathrm{ev}}$ are 2-element spinors satisfying $H_{\tau}\left(k_{x}, k_{y}\right) \chi_{\tau}=$ $\tau \epsilon \chi_{\tau}$ at $k_{x}=+k_{\tau}, k_{x}=-k_{\tau}$, and the evanescent wave vector $k_{x}=-i \kappa_{\tau}$, respectively. Here, $\chi_{\mathrm{i}, \mathrm{o}}$ can be normalized to 
$\|\chi\|^{2}=$ const., as they carry the same current. Then, $r_{\tau}$ is the reflection amplitude.

We note that $H_{\tau}\left(-k, k_{y}\right)=H_{\tau}\left(k, k_{y}\right)^{*}$ and that $H_{\tau}\left(-i \kappa, k_{y}\right)$ is real-valued, so that we can choose $\chi_{\mathrm{o}, \tau}=\chi_{\mathrm{i}, \tau}^{*}$ and $\chi_{\mathrm{ev}, \tau}$ is real.

The hard-wall boundary condition $\Psi_{\tau}(0)=0$ results to the reflection amplitude

$$
r_{\tau}=-\frac{\operatorname{det}\left(\chi_{\mathrm{i}, \tau}, \chi_{\mathrm{ev}, \tau}\right)}{\operatorname{det}\left(\chi_{\mathrm{o}, \tau}, \chi_{\mathrm{ev}, \tau}\right)}=-\frac{\operatorname{det}\left(\chi_{\mathrm{i}, \tau}, \chi_{\mathrm{ev}, \tau}\right)}{\operatorname{det}\left(\chi_{\mathrm{i}, \tau}, \chi_{\mathrm{ev}, \tau}\right)^{*}} .
$$

Hence, $r_{\tau}=-\exp \left[2 i \arg \operatorname{det}\left(\chi_{\mathrm{i}, \tau}, \chi_{\mathrm{ev}, \tau}\right)\right]$.

The quasiclassical reflection amplitude is evaluated at the Fermi surface, $\epsilon=0$. There, $k_{-}=k_{+}$and $\kappa_{-}=\kappa_{+}$. Using $H_{-}\left(k_{x}\right)=\sigma_{1} H_{+}\left(k_{x}^{*}\right)^{*} \sigma_{1}$, we can choose $\chi_{\mathrm{i},-}=\sigma_{1} \chi_{\mathrm{i},+}^{*}$ and $\chi_{\mathrm{ev},-}=\left.\sigma_{1} \chi_{\mathrm{ev},+}\right|_{-\kappa}$, where $\left.\chi_{\mathrm{ev},+}\right|_{-\kappa}$ is the real evanescent spinor at $k_{x}=+i \kappa$. Then, $r_{+} r_{-}^{*}=e^{2 i \theta}$ where

$$
\begin{gathered}
\theta=\arg z, \\
z=\operatorname{det}\left(\chi_{\mathrm{i},+}, \chi_{\mathrm{ev},+}\right) \operatorname{det}\left(\chi_{\mathrm{i},+},\left.\chi_{\mathrm{ev},+}\right|_{-\kappa}\right) .
\end{gathered}
$$

In the wave function basis used here (see App. A),

$$
\chi_{\mathrm{i},+} \propto\left(\begin{array}{c}
\alpha k_{F} e^{i \phi^{\prime} / 2} \\
\left(S_{F}+h_{z}\right) e^{-i \phi^{\prime} / 2}
\end{array}\right), \quad \chi_{\mathrm{ev},+} \propto\left(\begin{array}{c}
\alpha\left(k_{y}+\kappa\right) \\
S_{F}^{\prime}+h_{z}
\end{array}\right),
$$

where $\phi^{\prime}=\frac{\pi}{2}-\phi, \quad k_{F x}=k_{F} \sin \phi^{\prime}, \quad k_{y}=k_{F} \cos \phi^{\prime}, k_{F}^{2}=$ $2 m\left(S_{F}+\mu\right), \quad \kappa^{2}=k_{y}^{2}-2 m\left(S_{F}^{\prime}+\mu\right), \quad$ and $\quad S_{F}^{\prime}=2 m \alpha^{2}-$ $S_{F}=-S_{F}+\left(S_{F}^{2}-h_{z}^{2}\right) /\left(S_{F}+\mu\right)$. A mechanical (if long) calculation making these substitutions gives

$$
z=4 m \alpha^{2}\left(\mu-h_{z}\right) S_{F}\left(S_{F}-S_{F}^{\prime}\right)\left[\sin \phi-i \frac{h_{z}}{S_{F}} \cos \phi\right] .
$$

From this and Eq. (B3), we find Eq. (10).

For QAHI, Eq. (25), we can calculate the scattering phase change $\theta_{\mathrm{QAHI}}$ via the same method

$$
\theta_{\mathrm{QAHI}}=\arg \left[\left(1+\frac{B h_{z 2}}{D S_{F 2}}\right) \sin \phi-i\left(\frac{h_{z 2}}{S_{F 2}}+\frac{B}{D}\right) \cos \phi\right],
$$

where $h_{z 2}=B p_{F}^{2}+M$ and $S_{F 2}=\sqrt{h_{z 2}^{2}+A^{2} p_{F}^{2}}$.

\section{APPENDIX C: ZAITSEV'S BOUNDARY CONDITIONS}

Once the reflection matrix is known, we use the decoupling of the equations for the slowly varying quasiclassical parts from the fast-oscillating parts of the Green's function derived in Refs. [44,45]. Because the problem here involves a projection to the lower band, which complicates the discussion, we outline here for completeness how it can be handled. We also limit the discussion to the fully reflective interface, where the problem becomes simpler.

We consider the same setup as in Appendix B, with interface at $x=0$, but with Hamiltonian at $x<0$ slowly varying on a length scale $\lambda \gg \kappa^{-1}, k_{F x}^{-1}$. When $\left|x-x^{\prime}\right|,-x,-x^{\prime} \gg \kappa^{-1}$, the Green's function ansatz, for a fixed $k_{y}$, is

$$
\hat{G}_{1}\left(x, x^{\prime}\right)=\sum_{a b= \pm} e^{i k_{F x}\left(a x-b x^{\prime}\right)} \hat{C}_{a b}\left(x, x^{\prime}\right),
$$

$$
\hat{C}_{a b}\left(x, x^{\prime}\right)=\sum_{\tau, \tau^{\prime}= \pm}\left|\psi_{a,-, \tau}\right\rangle\left\langle\psi_{b,-, \tau^{\prime}}\right|\left(C_{a b}\right)_{\tau \tau^{\prime}}\left(x, x^{\prime}\right),
$$

where $C_{a b}\left(x, x^{\prime}\right)=\theta\left(x-x^{\prime}\right) C_{a b}^{>}\left(x, x^{\prime}\right)+\theta\left(x^{\prime}-x\right) C_{a b}^{<}\left(x, x^{\prime}\right)$ and $C_{a b}^{>/<}$are slowly varying amplitudes. Moreover, $\left|\psi_{a,-, \tau}\right\rangle$ are the lower-band null vectors, satisfying $\hat{H}_{0}\left(a k_{F x}, k_{y}\right)\left|\psi_{a,-, \tau}\right\rangle=$ 0 for the normal-state bulk Hamiltonian $H_{0}$ which is block diagonal in the Nambu index $\tau$.

Andreev approximation in the Gor'kov equation for $\hat{G}^{-1}=$ $\epsilon \tau_{3}-\hat{H}\left(x,-i \partial_{x}\right)$ with slowly varying $\hat{H}(x)$, and projection to the lower band gives, for $x \neq x^{\prime}$,

$$
\begin{aligned}
0 \simeq & \left\langle\psi_{a,-, \tau}\left|\left[\epsilon \tau_{3}-\hat{H}\left(x, a k_{F x}\right)\right] \hat{C}_{a b}\right| \psi_{b,-, \tau^{\prime}}\right\rangle \\
& +i\left\langle\psi_{a,-, \tau}\left|v_{x}\left(\partial_{x} \hat{C}_{a b}\right)\right| \psi_{b,-, \tau^{\prime}}\right\rangle \\
= & \left(\left[\epsilon \tau_{3}-\tilde{H}\left(x, a k_{F x}\right)\right] C_{a b}+i a v_{x} \partial_{x} C_{a b}\right)_{\tau \tau^{\prime}},
\end{aligned}
$$

and similarly for the adjoint equation,

$$
0 \simeq\left(C_{a b}\left[\epsilon \tau_{3}-\tilde{H}\left(x, b k_{F x}\right)\right]-i b \partial_{x^{\prime}} C_{a b} v_{x}\right)_{\tau \tau^{\prime}} .
$$

Here, $\quad\left(v_{x}\right)_{\tau \tau^{\prime}}=\left\langle\psi_{-, a, \tau}\left|\left(k_{F x} / m+a \alpha \sigma_{2}\right)\right| \psi_{-, a, \tau^{\prime}}\right\rangle=\delta_{\tau \tau^{\prime}}[1-$ $\left.\frac{m \alpha^{2}}{S_{F}}\right] \frac{k_{F x}}{m}=\delta_{\tau \tau^{\prime}} v_{F} \sin \phi^{\prime}$ is diagonal, and $\tilde{H}$ is the projected Hamiltonian. Hence, for $x$ away from the interface and $\lambda \gg$ $\delta \gg \kappa^{-1}, C_{++}(x, x \pm \delta), C_{--}(x, x \pm \delta)$ follow the quasiclassical Eilenberger equation.

When $\left|x-x^{\prime}\right| \gg \kappa^{-1}$, but either $x$ or $x^{\prime}$ is close to the interface at $x=0$, the evanescent state $\hat{H}_{0}\left(-i \kappa, k_{y}\right)\left|\psi_{\mathrm{ev}, \tau}\right\rangle=0$ also has a finite amplitude:

$$
\begin{gathered}
\hat{G}_{2}=\hat{G}_{1}+ \begin{cases}\sum_{b= \pm} e^{\kappa x-i b k_{F x} x^{\prime}} \hat{C}_{0 b}, & \text { for } x>x^{\prime}, \\
\sum_{a= \pm} e^{i a k_{F x} x+\kappa x^{\prime}} \hat{C}_{a 0}, & \text { for } x<x^{\prime},\end{cases} \\
\hat{C}_{a 0}=\sum_{\tau \tau^{\prime}}\left(C_{a 0}\right)_{\tau \tau^{\prime}}\left|\psi_{a,-, \tau}\right\rangle\left\langle\psi_{\mathrm{ev}, \tau^{\prime}}\right|, \\
\hat{C}_{0 b}=\sum_{\tau \tau^{\prime}}\left(C_{0 b}\right)_{\tau \tau^{\prime}}\left|\psi_{\mathrm{ev}, \tau}\right\rangle\left\langle\psi_{b,-, \tau^{\prime}}\right| .
\end{gathered}
$$

The ansatz by construction satisfies $\left(H_{0} G_{2}\right)\left(x, x^{\prime}\right)=$ $\left(G_{2} H_{0}\right)\left(x, x^{\prime}\right)=0$ when $C_{a b}^{>/<}$are constant. It satisfies also the boundary conditions $\hat{G}_{2}\left(0, x^{\prime}\right)=\hat{G}_{2}(x, 0)=0$ if

$$
\begin{aligned}
0= & \left(C_{+, b}^{>}\right)_{\tau \tau^{\prime}}\left|\psi_{+,-, \tau}\right\rangle+\left(C_{-, b}^{>}\right)_{\tau \tau^{\prime}}\left|\psi_{-,-, \tau}\right\rangle \\
& +\left(C_{0, b}^{>}\right)_{\tau \tau^{\prime}}\left|\psi_{\mathrm{ev}, \tau}\right\rangle, \\
0= & \left(C_{a,+}^{<}\right)_{\tau \tau^{\prime}}\left\langle\psi_{+,-, \tau^{\prime}}\right|+\left(C_{a,-}^{<}\right)_{\tau \tau^{\prime}}\left\langle\psi_{-,-, \tau^{\prime}}\right| \\
& +\left(C_{a, 0}^{<}\right)_{\tau \tau^{\prime}}\left\langle\psi_{\mathrm{ev}, \tau^{\prime}}\right| .
\end{aligned}
$$

This is the scattering problem solved in Appendix B above. The solution gives the boundary conditions $C_{++}^{>}=$ $\hat{R}^{\dagger} C_{-+}^{>}, C_{--}^{>}=\hat{R} C_{+-}^{>}, C_{++}^{<}=C_{+-}^{<} \hat{R}, C_{--}^{<}=C_{-+}^{<} \hat{R}^{\dagger}$ where $\hat{R}=\operatorname{diag}\left(r_{+}, r_{-}\right)$is the reflection matrix [Eq. (9)]. Note that the results here are more limited than in [45], as we assume the special case of a nontransparent and sharp interface, where the normal-state Hamiltonian stays constant up to the interface.

Writing the Green's function around $x=x^{\prime}$ also needs inclusion of additional terms $\propto e^{\mp \kappa\left(x-x^{\prime}\right)}$. The exact Green's function is continuous at $x=x^{\prime}$, with the jump condition $\left[\partial_{x} \hat{G}\right]_{x=x^{\prime}-0^{+}}^{x=0^{\prime}+0^{+}}=2 m$. For the ansatz at $x=x^{\prime}$, this implies continuity of the drone amplitudes, $C_{+-}^{<}(x, x)=C_{+-}^{>}(x, x)$, 
$C_{-+}^{<}(x, x)=C_{-+}^{>}(x, x)$, as they are the only components oscillating as $e^{ \pm 2 i k_{F x} x}$. The relations between $C_{a a}^{<}(x, x)$ and $C_{a a}^{>}(x, x)$ are more complicated, but are not necessary to find the reflective boundary condition. Together with the scattering boundary conditions, this implies that close to the interface (for $\lambda \gg|x|,\left|x^{\prime}\right| \gg \kappa^{-1}$ ), $C_{++}^{>}=\hat{R}^{\dagger} C_{--}^{<} \hat{R}, C_{++}^{<}=\hat{R}^{\dagger} C_{--}^{>} \hat{R}$.

The remaining problem is to relate $C_{a b}^{>1<}$ to the quasiclassical Green's function. To do this, we move Eq. (C1) to the Wigner representation assuming slowly varying $C_{a b}$, and drop the $\pm 2 k_{F x}$ drone amplitudes

$$
\hat{G}\left(k_{x} ; x\right) \simeq \sum_{a= \pm}\left(\frac{\hat{C}_{a a}^{>}(x, x)}{\eta-i\left(a k_{F x}-k_{x}\right)}+\frac{\hat{C}_{a a}^{<}(x, x)}{\eta+i\left(a k_{F x}-k_{x}\right)}\right),
$$

where $\eta \rightarrow 0^{+}$. The quasiclassical Green's function $\hat{g}$ is obtained by integrating over $\xi=v_{F}\left(k-k_{F}\right)=v_{F} \delta k$ in the vicinity of the Fermi surface after fixing the momentum direction so that $k_{x}=k \sin \phi^{\prime}$ and $k_{y}=k \cos \phi^{\prime}$. Because $k_{F x}=$ $\sqrt{k_{F}^{2}-k_{y}^{2}}=\sqrt{k_{F}^{2}-k^{2} \cos ^{2} \phi^{\prime}}$ also depends on $k$, linearizing around $k \approx k_{F}$ in (C11) gives

$$
a k_{F x}-k_{x} \simeq\left(a-a^{\prime}\right)\left(k_{F}+\delta k\right)\left|\sin \phi^{\prime}\right|-\frac{a \delta k}{\left|\sin \phi^{\prime}\right|},
$$

where $a^{\prime}=\operatorname{sgn} \sin \phi^{\prime}$. Hence, we have for $\hat{g}\left(x, \phi^{\prime}\right)$ :

$$
\begin{aligned}
\hat{g}\left(x, \phi^{\prime}\right) & =\frac{i}{\pi} \int v_{F} d\left(k-k_{F}\right) P_{-} \hat{G}\left(k_{x} ; x\right) P_{-}^{\dagger} \\
& \simeq i v_{F}\left|\sin \phi^{\prime}\right|\left[C_{a^{\prime} a^{\prime}}^{>}(x, x)+C_{a^{\prime} a^{\prime}}^{<}(x, x)\right],
\end{aligned}
$$

where $P_{-}$is the projector to the lower band, and only the $\delta$ function parts are included in the $\xi$-integration. The boundary conditions for $C_{a a}^{>/<}$now imply a Zaitsev boundary condition for $\hat{g}$ :

$$
\hat{g}\left(x=0,-\phi^{\prime}\right)=\hat{R} \hat{g}\left(x=0, \phi^{\prime}\right) \hat{R}^{\dagger},
$$

for $\sin \phi^{\prime}>0$, and we find Eq. (8).

The quasiclassical approach neglects a fast-oscillating part, which contributes a $\cos \left(2 k_{F} x\right)$ term in the DOS. However, we do not need to consider it in the problem with a single interface, as the equations for the slowly varying $\hat{g}$ are decoupled from the fast part. Similar decoupling was previously obtained from a different approach, explicitly for the 1D Majorana problem with a semiinfinite disordered bulk and a single interface [16]. However, interference effects, e.g., between multiple interfaces, are not captured in the quasiclassical approach [44]. This includes, e.g., the $k_{f} L$ oscillation of the energy level of overlapping Majorana end states [17].

\section{APPENDIX D: NUMERICAL CALCULATION}

We solve the Eilenberger equation numerically by using the simple iteration method. We first calculate the Green's function $\hat{g}_{1}$ in the absence of disorder. Then we substitute the disorder self-energy $\hat{\Sigma}_{1}=\hat{g}_{1} / \tau$ back into the Eilenberger equation and obtain another Green's function $\hat{g}_{2}$. We repeat this process several times until the difference between $\hat{g}_{n}$ and $\hat{g}_{n+1}$ is smaller than 0.001 .

\section{APPENDIX E: GAP CLOSING POINT}

In this Appendix, we show how to find the gap closing point for both $1 \mathrm{D}$ and $2 \mathrm{D}$ cases. We can write the bulk Green's function as $\hat{g}_{B}=g_{B, 1} \tilde{\tau}_{1}+g_{B, 2} \tilde{\tau}_{2}+g_{B, 3} \tilde{\tau}_{3}$, which is independent of position and momentum direction. In $1 \mathrm{D}, \hat{g}_{B}$ satisfies the Eilenberger equation

$$
\left[i \epsilon \tilde{\tau}_{3}+\Delta \tilde{\tau}_{1}+X^{2} \tilde{\tau}_{3} \hat{g}_{B} \tilde{\tau}_{3} / 2 \tau, \hat{g}_{B}\right]=0 .
$$

In $2 \mathrm{D}$, the bulk Eilenberger equation is given by

$$
\left[i \epsilon \tilde{\tau}_{3}+\Delta \tilde{\tau}_{1}+\hat{g}_{B} / 2 \tau+X^{2} \tilde{\tau}_{3} \hat{g}_{B} \tilde{\tau}_{3} / 2 \tau, \hat{g}_{B}\right]=0 .
$$

Note that Eq. (E1) is equivalent to Eq. (E2) because $\left[\hat{g}_{B} / 2 \tau, \hat{g}_{B}\right]=0$. Setting $\epsilon=0$, we get two solutions to Eq. (E1)

$$
g_{B, 2}=0, \quad g_{B, 3}=0, \quad g_{B, 1}=1,
$$

or

$g_{B, 2}=0, \quad g_{B, 3}=\sqrt{1-\Delta^{2} \tau^{2} / X^{4}}, \quad g_{B, 1}=\Delta \tau / X^{2}$.

These two solutions coincide at $1 / \tau=\Delta / X^{2}$. Making use of the "boundary conditions" $\hat{g}_{B}(1 / \tau=0)=\tilde{\tau}_{1}, \hat{g}_{B}(1 / \tau \rightarrow$ $+\infty)=\tilde{\tau}_{3}$ [42], we find the physical solution, which is for $1 / \tau<\Delta / X^{2}$

$$
g_{B, 2}=0, \quad g_{B, 3}=0, \quad g_{B, 1}=1,
$$

and for $1 / \tau<\Delta / X^{2}$

$$
g_{B, 2}=0, \quad g_{B, 3}=\sqrt{1-\Delta^{2} \tau^{2} / X^{4}}, \quad g_{B, 1}=\Delta \tau / X^{2} .
$$

Therefore the gap closing point is $1 / \tau=\Delta / X^{2}$.

\section{APPENDIX F: MAJORANA LOCALIZATION LENGTH NEAR THE GAP CLOSING POINT}

\section{One-dimensional case}

In one dimension, the Eilenberger equation is given by

$$
v_{F} \nabla \hat{g}_{\lambda}=\left[\hat{g}_{\lambda}, i \epsilon \tilde{\tau}_{3}+\Delta \tilde{\tau}_{1}+\hat{\Sigma}_{\lambda}\right],
$$

where $\lambda=+/-$ corresponds to right/left going electrons. The disorder self-energy is given by $\hat{\Sigma}_{\lambda}=X^{2} \tilde{\tau}_{3} \hat{g}_{-\lambda} \tilde{\tau}_{3} / 2 \tau$. For convenience, we write the Green's function as

$$
\hat{g}_{\lambda}=\hat{g}_{B, \lambda}+\hat{g}_{E, \lambda},
$$

where $\hat{g}_{B, \lambda}$ and $\hat{g}_{E, \lambda}$ are bulk and edge Green's functions, respectively. Far away from the boundary, $\hat{g}_{E}$ is much smaller than $\hat{g}_{B}$ and can be treated as a perturbation. Thus, we can expand Eq. (F1) up to the first order in $\hat{g}_{E}$. The 0th order terms are gone because they just give the bulk Eilenberger equation. The first-order terms are given by

$$
\begin{aligned}
v_{F} \nabla \hat{g}_{E, \lambda}= & {\left[\hat{g}_{E, \lambda}, \Delta \tilde{\tau}_{1}+X^{2} \tilde{\tau}_{3} \hat{g}_{B,-\lambda} \tilde{\tau}_{3} / 2 \tau\right] } \\
& +\left[\hat{g}_{B, \lambda}, X^{2} \tilde{\tau}_{3} \hat{g}_{E,-\lambda} \tilde{\tau}_{3} / 2 \tau\right] .
\end{aligned}
$$

Here we have already set $\epsilon=0$. Before the gap closes the bulk Green's function is just $\hat{g}_{B, \lambda}=\tilde{\tau}_{1}$. We also notice that $\hat{g}_{E,+}$ and $\hat{g}_{E,-}$ have the relation $\hat{g}_{E,+, 1}=\hat{g}_{E,-, 1}, \hat{g}_{E,+, 2}=-\hat{g}_{E,-, 2}$, $\hat{g}_{E,+, 3}=\hat{g}_{E,-, 3}$ for the components $\hat{g}_{E, \lambda}=\sum_{j=1}^{3} \hat{g}_{E, \lambda, j} \tilde{\tau}_{j}$. 
Thus Eq. (F3) can be simplified as

$$
v_{F} \nabla \hat{g}_{E, \lambda}=\left[\hat{g}_{E, \lambda},\left(\Delta-X^{2} / \tau\right) \tilde{\tau}_{1}\right] .
$$

It can be seen that the effective pairing is reduced to $\Delta-$ $X^{2} / \tau$. The Majorana localization length is given by $l_{M}=$ $v_{F} /\left(\Delta-X^{2} / \tau\right)$, which is divergent at the gap closing point $1 / \tau=\Delta / X^{2}$. Our result is at odds with the numerical study in Ref. [16], which finds $l_{M}=v_{F}(\Delta-1 / \tau)^{-0.84}$. However, it is consistent with the result from the transfer matrix method in Ref. [28] despite the fact that this paper disregards the edge contribution to the self-energy for strong disorder in the Born approximation approach.

\section{Two-dimensional case}

In two dimensions, the Eilenberger equation is given by Eq. (5). Using the same method as in the one-dimensional case, we arrive at

$$
v_{F} \nabla \hat{g}_{E,+}=\left[\hat{g}_{E,+},\left(\Delta+1 / 2 \tau-X^{2} / 2 \tau\right) \tilde{\tau}_{1}\right]+\left[\tilde{\tau}_{1}, \hat{\Sigma}_{E}\right],
$$

where $\hat{g}_{E,+}$ is the edge Green's function with relative momentum pointing to the positive $x$ direction. If we would assume $\hat{\Sigma}_{E}=\left(1+X^{2}\right) \hat{g}_{E,+} / 2 \tau$, Eq. (F5) would be simplified as

$$
v_{F} \nabla \hat{g}_{E,+}=\left[\hat{g}_{E,+},\left(\Delta-X^{2} / \tau\right) \tilde{\tau}_{1}\right] .
$$

Equation (F6) is almost the same as Eq. (F4). At the gap closing point $1 / \tau=\Delta / X^{2}$, the effective pairing is reduced to 0 and the Majorana localization length diverges. However, in practice $\hat{\Sigma}_{E}$ is smaller than $\left(1+X^{2}\right) \hat{g}_{E,+} / 2 \tau$ and is not large enough to reduce the effective pairing to zero. Therefore the Majorana localization length is finite at the gap closing point.
[1] X.-L. Qi and S.-C. Zhang, Rev. Mod. Phys. 83, 1057 (2011).

[2] A. Y. Kitaev, Phys. Usp. 44, 131 (2001).

[3] L. Fu and C. L. Kane, Phys. Rev. Lett. 100, 096407 (2008).

[4] J. D. Sau, R. M. Lutchyn, S. Tewari, and S. Das Sarma, Phys. Rev. Lett. 104, 040502 (2010).

[5] A. Yamakage, Y. Tanaka, and N. Nagaosa, Phys. Rev. Lett. 108, 087003 (2012).

[6] K. T. Law, P. A. Lee, and T. K. Ng, Phys. Rev. Lett. 103, 237001 (2009).

[7] V. Mourik, K. Zuo, S. M. Frolov, S. Plissard, E. P. Bakkers, and L. P. Kouwenhoven, Science 336, 1003 (2012).

[8] S. Nadj-Perge, I. K. Drozdov, J. Li, H. Chen, S. Jeon, J. Seo, A. H. MacDonald, B. A. Bernevig, and A. Yazdani, Science 346, 602 (2014).

[9] Q. L. He, L. Pan, A. L. Stern, E. C. Burks, X. Che, G. Yin, J. Wang, B. Lian, Q. Zhou, E. S. Choi et al., Science 357, 294 (2017).

[10] O. Motrunich, K. Damle, and D. A. Huse, Phys. Rev. B 63, 224204 (2001).

[11] P. W. Brouwer, M. Duckheim, A. Romito, and F. vonOppen, Phys. Rev. Lett. 107, 196804 (2011).

[12] A. M. Lobos, R. M. Lutchyn, and S. DasSarma, Phys. Rev. Lett. 109, 146403 (2012).

[13] F. Pientka, A. Romito, M. Duckheim, Y. Oreg, and F. von Oppen, New J. Phys. 15, 025001 (2013).

[14] D. A. Huse, R. Nandkishore, V. Oganesyan, A. Pal, and S. L. Sondhi, Phys. Rev. B 88, 014206 (2013).

[15] İ. Adagideli, M. Wimmer, and A. Teker, Phys. Rev. B 89, 144506 (2014).

[16] H.-Y. Hui, J. D. Sau, and S. Das Sarma, Phys. Rev. B 90, 064516 (2014).

[17] V. Stanev and V. Galitski, Phys. Rev. B 89, 174521 (2014).

[18] A. C. Potter and P. A. Lee, Phys. Rev. Lett. 105, 227003 (2010).

[19] M.-T. Rieder, P. W. Brouwer, and I. Adagideli, Phys. Rev. B 88, 060509(R) (2013).

[20] M.-T. Rieder and P. W. Brouwer, Phys. Rev. B 90, 205404 (2014).

[21] B. Lu, P. Burset, Y. Tanuma, A. A. Golubov, Y. Asano, and Y. Tanaka, Phys. Rev. B 94, 014504 (2016).
[22] P. Burset, B. Lu, S. Tamura, and Y. Tanaka, Phys. Rev. B 95, 224502 (2017).

[23] M. Hell, M. Leijnse, and K. Flensberg, Phys. Rev. Lett. 118, 107701 (2017).

[24] F. Pientka, A. Keselman, E. Berg, A. Yacoby, A. Stern, and B. I. Halperin, Phys. Rev. X 7, 021032 (2017).

[25] M. Hell, K. Flensberg, and M. Leijnse, Phys. Rev. B 96, 035444 (2017).

[26] S. Hart, H. Ren, M. Kosowsky, G. Ben-Shach, P. Leubner, C. Brüne, H. Buhmann, L. W. Molenkamp, B. I. Halperin, and A. Yacoby, Nat. Phys. 13, 87 (2017).

[27] S. Głodzik, N. Sedlmayr, and T. Domański, Phys. Rev. B 102, 085411 (2020).

[28] A. Haim and A. Stern, Phys. Rev. Lett. 122, 126801 (2019).

[29] X.-L. Qi, T. L. Hughes, and S.-C. Zhang, Phys. Rev. B 82, 184516 (2010).

[30] S. B. Chung, X.-L. Qi, J. Maciejko, and S.-C. Zhang, Phys. Rev. B 83, 100512(R) (2011).

[31] J. Wang, Q. Zhou, B. Lian, and S.-C. Zhang, Phys. Rev. B 92, 064520 (2015).

[32] S.-I. Suzuki, A. A. Golubov, Y. Asano, and Y. Tanaka, Phys. Rev. B 100, 024511 (2019).

[33] Y. Asano and Y. Tanaka, Phys. Rev. B 87, 104513 (2013).

[34] Y. Tanaka, M. Sato, and N. Nagaosa, J. Phys. Soc. Jpn. 81, 011013 (2011).

[35] Y. Tanaka and A. A. Golubov, Phys. Rev. Lett. 98, 037003 (2007).

[36] Y. Tanaka and S. Kashiwaya, Phys. Rev. B 70, 012507 (2004).

[37] Y. Tsutsumi and K. Machida, Phys. Rev. B 85, 100506(R) (2012).

[38] S.-I. Suzuki and Y. Asano, Phys. Rev. B 94, 155302 (2016).

[39] S. V. Bakurskiy, A. A. Golubov, M. Y. Kupriyanov, K. Yada, and Y. Tanaka, Phys. Rev. B 90, 064513 (2014).

[40] J. Sauls, Phys. Rev. B 84, 214509 (2011).

[41] G. Eilenberger, Z. Phys. A 214, 195 (1968).

[42] Y. Lu and T. T. Heikkilä, Phys. Rev. B 100, 104514 (2019).

[43] A. Zyuzin, M. Alidoust, and D. Loss, Phys. Rev. B 93, 214502 (2016). 
[44] A. V. Zaitsev, Zh. Eksp. Teor. Fiz. 86, 1742 (1984).

[45] A. Millis, D. Rainer, and J. A. Sauls, Phys. Rev. B 38, 4504 (1988).

[46] F. Evers and A. D. Mirlin, Rev. Mod. Phys. 80, 1355 (2008).
[47] A. Mildenberger, F. Evers, A. D. Mirlin, and J. T. Chalker, Phys. Rev. B 75, 245321 (2007).

[48] Y. Zhang, K. He, C.-Z. Chang, C.-L. Song, L.-L. Wang, X. Chen, J.-F. Jia, Z. Fang, X. Dai, W.-Y. Shan et al., Nat. Phys. 6, 584 (2010). 